JOURNAL OF THE

AMERICAN MATHEMATICAL SOCIETY

Volume 13, Number 3, Pages 467-479

S 0894-0347(00)00331-3

Article electronically published on March 13, 2000

\title{
SEMI-INVARIANTS OF QUIVERS AND SATURATION FOR LITTLEWOOD-RICHARDSON COEFFICIENTS
}

\author{
HARM DERKSEN AND JERZY WEYMAN
}

\section{INTRODUCTION}

Let $Q$ be a quiver without oriented cycles. Let $\alpha$ be a dimension vector for $Q$. We denote by $\operatorname{SI}(Q, \alpha)$ the ring of semi-invariants of the set of $\alpha$-dimensional representations of $Q$ over a fixed algebraically closed field $K$.

In this paper we prove some results about the set

$$
\Sigma(Q, \alpha)=\left\{\sigma \mid \operatorname{SI}(Q, \alpha)_{\sigma} \neq 0\right\} .
$$

$\Sigma(Q, \alpha)$ is defined in the space of all weights by one homogeneous linear equation and by a finite set of homogeneous linear inequalities. In particular the set $\Sigma(Q, \alpha)$ is saturated, i.e., if $n \sigma \in \Sigma(Q, \alpha)$, then also $\sigma \in \Sigma(Q, \alpha)$.

These results, when applied to a special quiver $Q=T_{n, n, n}$ and to a special dimension vector, show that the $\mathrm{GL}_{n}$-module $V_{\lambda}$ appears in $V_{\mu} \otimes V_{\nu}$ if and only if the partitions $\lambda, \mu$ and $\nu$ satisfy an explicit set of inequalities. This gives new proofs of the results of Klyachko $([7,3])$ and Knutson and Tao ([8]).

The proof is based on another general result about semi-invariants of quivers (Theorem 11). In the paper [10, Schofield defined a semi-invariant $c_{W}$ for each indecomposable representation $W$ of $Q$. We show that the semi-invariants of this type span each weight space in $\operatorname{SI}(Q, \alpha)$. This seems to be a fundamental fact, connecting semi-invariants and modules in a direct way. Given this fact, the results on sets of weights follow at once from the results in another paper of Schofield [11.

\section{THE RESUlts}

A quiver $Q$ is a pair $Q=\left(Q_{0}, Q_{1}\right)$ consisting of the set of vertices $Q_{0}$ and the set of arrows $Q_{1}$. Each arrow $a$ has its head $h a$ and tail $t a$, both in $Q_{0}$ :

$$
t a \stackrel{a}{\longrightarrow} h a .
$$

We fix an algebraically closed field $K$. A representation (or a module) $V$ of $Q$ is a family of finite dimensional vector spaces $\left\{V(x) \mid x \in Q_{0}\right\}$ and of linear maps

Received by the editors July 20, 1999.

2000 Mathematics Subject Classification. Primary 13A50; Secondary 14L24, 14L30, 16G20, $20 \mathrm{G} 05$.

Key words and phrases. Quiver representations, semi-invariants, Littlewood-Richardson coefficients, Klyachko cone, saturation.

The second author was supported by NSF, grant DMS 9700884 and KBN No. PO3A 01214. 
$V(a): V(t a) \rightarrow V(h a)$. The dimension vector of a representation $V$ is the function $\underline{d}(V): Q_{0} \rightarrow \mathbb{Z}_{\geq 0}$ defined by $\underline{d}(V)(x):=\operatorname{dim} V(x)$. The dimension vectors lie in the space $\Gamma$ of integer-valued functions on $Q_{0}$. A morphism $\phi: V \rightarrow V^{\prime}$ of two representations is a collection of linear maps $\phi(x): V(x) \rightarrow V^{\prime}(x), x \in Q_{0}$, such that for each $a \in Q_{1}$ we have $\phi(h a) V(a)=V^{\prime}(a) \phi(t a)$. We denote the linear space of morphisms from $V$ to $V^{\prime}$ by $\operatorname{Hom}_{Q}\left(V, V^{\prime}\right)$.

A path $p$ in $Q$ is a sequence of arrows $p=a_{1}, \ldots, a_{n}$ such that $h a_{i}=t a_{i+1}$ $(1 \leq i \leq n-1)$. We define $t p=t a_{1}, h p=h a_{n}$. We also have the trivial path $e(x)$ from $x$ to $x$. If $V$ is a representation and $p=a_{1}, \ldots, a_{n}$, then we define $V(p):=V\left(a_{n}\right) V\left(a_{n-1}\right) \cdots V\left(a_{1}\right)$. We assume throughout the paper that $Q$ has no oriented cycles, i.e., there are no paths $p=a_{1}, \ldots, a_{n}$ such that $t a_{1}=h a_{n}$.

For representations $V$ and $W$ of $Q$ there is a canonical exact sequence ([9])

$$
\begin{aligned}
0 \rightarrow \operatorname{Hom}_{Q}(V, W) \stackrel{i}{\rightarrow} \bigoplus_{x \in Q_{0}} \operatorname{Hom}(V(x), W(x)) \\
\qquad \stackrel{d_{W}^{V}}{\longrightarrow} \bigoplus_{a \in Q_{1}} \operatorname{Hom}(V(t a), W(h a)) \stackrel{p}{\rightarrow} \operatorname{Ext}_{Q}(V, W) \rightarrow 0 .
\end{aligned}
$$

The map $i$ is the obvious inclusion, the map $d_{W}^{V}$ is given by

$$
\{f(x)\}_{x \in Q_{0}} \mapsto\{f(h a) V(a)-W(a) f(t a)\}_{a \in Q_{1}},
$$

and the map $p$ constructs an extension of the representations $V$ and $W$ by adding the maps $V(t a) \rightarrow W(h a)$ to the direct sum representation $V \oplus W$.

For $\alpha, \beta \in \Gamma$ we define the Euler inner product

$$
\langle\alpha, \beta\rangle=\sum_{x \in Q_{0}} \alpha(x) \beta(x)-\sum_{a \in Q_{1}} \alpha(t a) \beta(h a) .
$$

It follows from (1) that $\langle\underline{d}(V), \underline{d}(W)\rangle=\operatorname{dim}_{K} \operatorname{Hom}_{Q}(V, W)-\operatorname{dim}_{K} \operatorname{Ext}_{Q}(V, W)$.

For a dimension vector $\alpha$ we denote by

$$
\operatorname{Rep}(Q, \alpha):=\bigoplus_{a \in Q_{1}} \operatorname{Hom}\left(K^{\alpha(t a)}, K^{\alpha(h a)}\right)
$$

the vector space of $\alpha$-dimensional representations of $Q$. The group

$$
\operatorname{GL}(Q, \alpha):=\prod_{x \in Q_{0}} \operatorname{GL}(\alpha(x))
$$

and its subgroup

$$
\operatorname{SL}(Q, \alpha)=\prod_{x \in Q_{0}} \operatorname{SL}(\alpha(x))
$$

act on $\operatorname{Rep}(Q, \alpha)$ in an obvious way. We are interested in the ring of semi-invariants

$$
\operatorname{SI}(Q, \alpha):=K[\operatorname{Rep}(Q, \alpha)]^{\operatorname{SL}(Q, \alpha)} .
$$


The ring $\operatorname{SI}(Q, \alpha)$ has a weight space decomposition

$$
\mathrm{SI}(Q, \alpha)=\bigoplus_{\sigma} \mathrm{SI}(Q, \alpha)_{\sigma}
$$

where $\sigma$ runs through the (one-dimensional irreducible) characters of $\operatorname{GL}(Q, \alpha)$ and

$$
\operatorname{SI}(Q, \alpha)_{\sigma}=\{f \in K[\operatorname{Rep}(Q, \alpha)] \mid g(f)=\sigma(g) f \forall g \in \operatorname{GL}(Q, \alpha)\} .
$$

Suppose that $\sigma$ lies in the dual space $\Gamma^{\star}:=\operatorname{Hom}(\Gamma, \mathbb{Z})$. For each dimension vector $\alpha$ we can associate to $\sigma$ a character of $\operatorname{GL}(Q, \alpha)$ defined as

$$
\prod_{x \in Q_{0}} d_{x}^{\sigma\left(e_{x}\right)}
$$

where $d_{x}$ is the determinant function on $\operatorname{GL}(\alpha(x))$ and $e_{x}$ is the dimension vector defined by

$$
e_{x}(y)= \begin{cases}1 & \text { if } x=y \\ 0 & \text { otherwise }\end{cases}
$$

In this way we will identify characters with $\Gamma^{\star}$. Sometimes, for convenience, we will write $\sigma(x)$ instead of $\sigma\left(e_{x}\right)$ (and treat $\sigma$ as an element of $\Gamma$ ).

Let us choose the dimension vectors $\alpha$ and $\beta$ in such way that $\langle\alpha, \beta\rangle=0$. Then for every $V \in \operatorname{Rep}(Q, \alpha)$ and $W \in \operatorname{Rep}(Q, \beta)$ the matrix of $d_{W}^{V}$ will be a square matrix. Following [10] we can therefore define the semi-invariant $c$ of the action of $\operatorname{GL}(Q, \alpha) \times \operatorname{GL}(Q, \beta)$ on $\operatorname{Rep}(Q, \alpha) \times \operatorname{Rep}(Q, \beta)$ by $c(V, W):=\operatorname{det} d_{W}^{V}$. The value of the determinant depends on the choices of bases, so $c$ is well-defined up to a scalar. Notice that the semi-invariant $c$ vanishes at the point $(V, W)$ if and only if $\operatorname{Hom}_{Q}(V, W) \neq 0$ which is equivalent to $\operatorname{Ext}_{Q}(V, W) \neq 0$. For a fixed $V$ the restriction of $c$ to $\{V\} \times \operatorname{Rep}(Q, \beta)$ defines a semi-invariant $c^{V}$ in $\operatorname{SI}(Q, \beta)$. Schofield proves ([10, Lemma 1.4]) that the weight of $c^{V}$ equals $\langle\alpha, \cdot\rangle \in \Gamma^{\star}$ which is defined as $\gamma \mapsto\langle\alpha, \gamma\rangle$. Similarly, for a fixed $W$ the restriction of $c$ to $\operatorname{Rep}(Q, \alpha) \times\{W\}$ defines a semi-invariant $c_{W}$ in $\operatorname{SI}(Q, \alpha)$ of weight $-\langle\cdot, \beta\rangle$ ([10, Lemma 1.4]). If $V, V^{\prime} \in \operatorname{Rep}(Q, \alpha)$ and $V \cong V^{\prime}$, then $V$ and $V^{\prime}$ are in the same $\operatorname{GL}(Q, \alpha)$-orbit, and $c^{V}$ and $c^{V^{\prime}}$ are equal up to a constant scalar. Semi-invariants of the types $c^{V}$ and $c_{W}$ are well-defined up to a scalar. These semi-invariants have the following properties.

Lemma 1. Suppose that $V, V^{\prime}, V^{\prime \prime}$ and $W, W^{\prime}, W^{\prime \prime}$ are representations of $Q$ such that $\langle\underline{d}(V), \underline{d}(W)\rangle=0$, and that there are exact sequences

$$
0 \rightarrow V^{\prime} \rightarrow V \rightarrow V^{\prime \prime} \rightarrow 0, \quad 0 \rightarrow W^{\prime} \rightarrow W \rightarrow W^{\prime \prime} \rightarrow 0
$$

a) If $\left\langle\underline{d}\left(V^{\prime}\right), \underline{d}(W)\right\rangle<0$, then $c^{V}(W)=0$;

b) If $\left\langle\underline{d}\left(V^{\prime}\right), \underline{d}(W)\right\rangle=0$, then $c^{V}(W)=c^{V^{\prime}}(W) c^{V^{\prime \prime}}(W)$;

c) If $\left\langle\underline{d}(V), \underline{d}\left(W^{\prime}\right)\right\rangle>0$, then $c^{V}(W)=0$;

d) If $\left\langle\underline{d}(V), \underline{d}\left(W^{\prime}\right)\right\rangle=0$, then $c^{V}(W)=c^{V}\left(W^{\prime}\right) c^{V}\left(W^{\prime \prime}\right)$. 
Proof. Consider the following commutative diagram with exact columns:

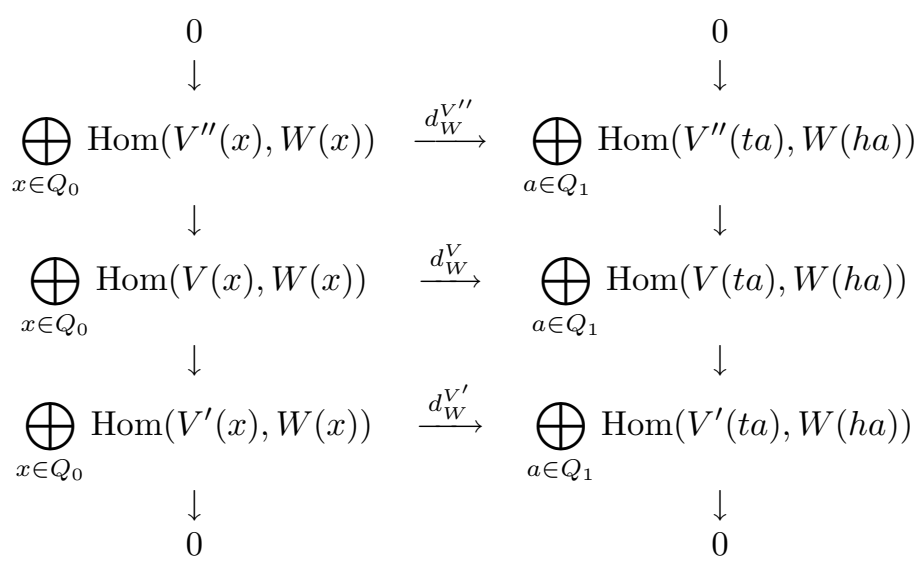

If $\left\langle\underline{d}\left(V^{\prime}\right), \underline{d}(W)\right\rangle=0$, then $d_{W}^{V^{\prime}}, d_{W}^{V}$ and $d_{W}^{V^{\prime \prime}}$ are all represented by square matrices. It follows that $c^{V}(W)=c^{V^{\prime}}(W) c^{V^{\prime \prime}}(W)$. So b) follows and d) goes similarly. If $\left\langle\underline{d}\left(V^{\prime}\right), \underline{d}(W)\right\rangle<0$, then $d_{W}^{V^{\prime}}$ cannot be surjective, hence $d_{W}^{V}$ is not surjective. Now a) follows and c) goes similarly.

Our main result is that the semi-invariants of type $c^{V}$ (resp. $c_{W}$ ) span all the weight spaces in the rings $\operatorname{SI}(Q, \alpha)$.

Theorem 1. Let $Q$ be a quiver without oriented cycles and let $\beta$ be a dimension vector. The ring of semi-invariants $\operatorname{SI}(Q, \beta)$ is a $K$-linear span of semi-invariants $c^{V}$ with $\langle\underline{d}(V), \beta\rangle=0$. The analogous result is true for the semi-invariants $c_{W}$.

After this paper was submitted we learned about the paper 12 where among other things the authors give another proof of Theorem 1 under the assumption that the characteristic of $K$ is zero.

We will prove Theorem 1 in Section 4.

Remark 1. If $V=V_{1} \oplus V_{2}$ is decomposable, then by Lemma 1 we have $c^{V}=0$ if $\left\langle\underline{d}\left(V_{1}\right), \beta\right\rangle \neq 0$, and $c^{V}=c^{V_{1}} c^{V_{2}}$ if $\left\langle\underline{d}\left(V_{1}\right), \beta\right\rangle=0$.

The algebra $\operatorname{SI}(Q, \beta)$ is generated by all $c^{V}$ where $V$ is indecomposable. Generators of $\operatorname{SI}(Q, \beta)$ therefore can be found in the degrees $\langle\alpha, \cdot\rangle$ such that a general representation of dimension $\alpha$ is indecomposable. By [5] this is equivalent to $\alpha$ being a Schur root.

Remark 2. If $\operatorname{Rep}(Q, \beta)$ has a dense $\operatorname{GL}(Q, \beta)$-orbit, then Schofield showed in [10] that the invariants of type $c^{V}$ with $V$ indecomposable generate $\operatorname{SI}(Q, \beta)$ (which is a polynomial ring in this case).

Theorem 1 has the following remarkable consequence.

Corollary 1 (Reciprocity Property). Let $\alpha, \beta$ be two dimension vectors for the quiver $Q$. Assume that $\langle\alpha, \beta\rangle=0$. Then

$$
\operatorname{dim}_{K} \operatorname{SI}(Q, \beta)_{\langle\alpha, \cdot\rangle}=\operatorname{dim}_{K} \operatorname{SI}(Q, \alpha)_{-\langle\cdot, \beta\rangle} \cdot
$$

Proof. Let $V_{1}, \ldots, V_{s}$ be the modules of dimension $\alpha$ such that $c^{V_{1}}, \ldots, c^{V_{s}}$ form a basis of $\operatorname{SI}(Q, \beta)_{\langle\alpha, \cdot\rangle}$. These are linearly independent polynomials on $\operatorname{Rep}(Q, \beta)$ so there exist $s$ representations $W_{1}, \ldots, W_{s}$ in $\operatorname{Rep}(Q, \beta)$ such that $\operatorname{det}\left(c^{V_{i}}\left(W_{j}\right)\right)_{1 \leq i, j \leq s}$ 
is not zero. But $c^{V_{i}}\left(W_{j}\right)=c_{W_{j}}\left(V_{i}\right)$ and this means that the semi-invariants $c_{W_{1}}, \ldots, c_{W_{s}}$ are linearly independent. This proves that

$$
\operatorname{dim}_{K} \operatorname{SI}(Q, \beta)_{\langle\alpha, \cdot\rangle} \leq \operatorname{dim}_{K} \operatorname{SI}(Q, \alpha)_{-\langle\cdot, \beta\rangle} \cdot
$$

The other inequality is proven in exactly the same way.

In the remainder of this section we investigate the consequences of Theorem 1 First we recall the main results of 11. They can be summarized as follows.

We say that for two dimension vectors $\alpha, \beta$ the space $\operatorname{Hom}_{Q}(\alpha, \beta)$ (respectively $\operatorname{Ext}_{Q}(\alpha, \beta)$ ) vanishes generically if and only if for general representations $V, W$ of dimensions $\alpha, \beta$ respectively we have $\operatorname{Hom}_{Q}(V, W)=0\left(\operatorname{resp} \operatorname{Ext}_{Q}(V, W)=0\right)$. We also write $\alpha \hookrightarrow \beta$ if a general representation of dimension $\beta$ has a subrepresentation of dimension $\alpha$.

Theorem 2 (Schofield). Let $\alpha$ and $\beta$ be two dimension vectors for the quiver $Q$.

a) $\operatorname{Ext}_{Q}(\alpha, \beta)$ vanishes generically if and only if $\alpha \hookrightarrow \alpha+\beta$,

b) $\operatorname{Ext}_{Q}(\alpha, \beta)$ does not vanish generically if and only if $\beta^{\prime} \hookrightarrow \beta$ and $\left\langle\alpha, \beta-\beta^{\prime}\right\rangle<0$ for some dimension vector $\beta^{\prime}$.

Part a) is proven in Section 3 of [11], and part b) is proven in Section 5.

Remark 3. Suppose that $V$ and $W$ are general modules of dimension $\alpha$ and $\beta$ respectively, such that $\langle\alpha, \beta\rangle=0$. The condition in b) is equivalent to $\exists \beta^{\prime} \beta^{\prime} \hookrightarrow \beta$ such that $\left\langle\alpha, \beta^{\prime}\right\rangle>0$. If $c^{V}(W)=0$, then $W$ must have a submodule $W^{\prime}$ such that $\left\langle\alpha, \underline{d}\left(W^{\prime}\right)\right\rangle>0$. This means that the converse of Lemma 1 $\left.1 \mathrm{c}\right)$ is true for general $V$ and $W$.

Theorem 3. Let $Q$ be a quiver without oriented cycles and let $\beta$ be a dimension vector. The semigroup $\Sigma(Q, \beta)$ is the set of all $\sigma \in \Gamma$ such that $\sigma(\beta)=0$ and $\sigma\left(\beta^{\prime}\right) \leq 0$ for all $\beta^{\prime}$ such that $\beta^{\prime} \hookrightarrow \beta$. Thus this condition is provided by one linear homogeneous equality and finitely many linear homogeneous inequalities. In particular the set $\Sigma(Q, \beta)$ is saturated in the lattice $\Gamma$.

Proof. Suppose that $\sigma \in \Gamma^{\star}$. We can write $\sigma=\langle\alpha, \cdot\rangle$ with $\alpha \in \Gamma$.

We will first assume that $\alpha$ is a dimension vector, i.e., $\alpha(x) \geq 0$ for all $x \in Q_{0}$. It follows from Theorem 1 that $\operatorname{SI}(Q, \beta)_{\langle\alpha, \cdot\rangle}$ is non-zero if and only if there exists a representation $V$ of dimension $\alpha$ such that $c^{V}$ is not zero, which is equivalent to $\sigma(\beta)=\langle\alpha, \beta\rangle=0$ and $\operatorname{Ext}_{Q}(\alpha, \beta)$ vanishing generically. By part b) of Theorem 2. $\operatorname{Ext}_{Q}(\alpha, \beta)$ vanishes generically if and only if for all $\beta^{\prime}$ such that $\beta^{\prime} \hookrightarrow \beta$ we have $\left\langle\alpha, \beta-\beta^{\prime}\right\rangle \geq 0$. This means that for all $\beta^{\prime}$ such that $\beta^{\prime} \hookrightarrow \beta$ we have $\sigma\left(\beta^{\prime}\right)=\left\langle\alpha, \beta^{\prime}\right\rangle \leq 0$. We conclude that $\operatorname{SI}(Q, \beta)_{\sigma} \neq 0$ if and only if $\sigma(\beta)=0$ and $\sigma\left(\beta^{\prime}\right) \leq 0$ for all $\beta^{\prime} \hookrightarrow \beta$.

If $\alpha$ is not a dimension vector, then $\operatorname{SI}(Q, \beta)_{n \sigma}=0$ for all integers $n>0$. Suppose that $W \in \operatorname{Rep}(Q, \beta)$. From [ 6 ] it follows that either $\sigma(\underline{d}(W)) \neq 0$ or there exists a submodule $W^{\prime}$ of $W$ such that $\sigma\left(\underline{d}\left(W^{\prime}\right)\right)>0$. If $W$ is in general position, then we obtain $\sigma(\beta) \neq 0$ or $\sigma\left(\beta^{\prime}\right)>0$ for some $\beta^{\prime} \hookrightarrow \beta$ (see also Remark 5 ).

Remark 4. Schofield in [11 gives an algorithm allowing one to determine the set of inequalities in Theorem 3 inductively. This algorithm is not very efficient.

Remark 5. A module $W \in \operatorname{Rep}(Q, \beta)$ is called $\sigma$-stable if and only if there exist an $n>0$ and an $f \in \operatorname{SI}(Q, \beta)_{n \sigma}$ such that $f(W) \neq 0$. King proved in [6] that a module 
$W \in \operatorname{Rep}(Q, \beta)$ is $\sigma$-stable if and only if $\sigma\left(W^{\prime}\right) \leq 0$ for all submodules $W^{\prime}$ of $W$. Applied to a general representation $W$ of dimension $\beta$ this gives us the equivalence:

$$
\exists n>0 \operatorname{SI}(Q, \beta)_{n \sigma} \neq 0 \Leftrightarrow \sigma(\beta)=0 \text { and } \forall \beta^{\prime} \beta^{\prime} \hookrightarrow \beta \text { we have } \sigma\left(\beta^{\prime}\right) \leq 0 .
$$

This shows that the saturation of $\Sigma(Q, \beta)$ is given by linear inequalities but it does not show that $\Sigma(Q, \beta)$ is saturated.

Remark 6 . In Theorem 3 instead of considering all $\beta^{\prime}$ with $\beta^{\prime} \hookrightarrow \beta$ we only need to consider those $\beta^{\prime}$ such that the general representation of dimension $\beta^{\prime}$ is indecomposable, which is equivalent to $\beta^{\prime}$ being a Schur root. Still, the set of inequalities obtained in this way may not be a minimal set of inequalities as we will see in the next example.

Example 1. Let $Q$ be the quiver

$$
\begin{array}{r}
1 \\
\downarrow \\
\downarrow \\
5 \\
5 \\
\uparrow \\
3
\end{array}
$$

and let $\beta$ be the dimension vector

$$
\begin{array}{lll} 
& 1 & \\
1 & 2 & 1 \\
& 1 &
\end{array} .
$$

For a general representation $V$ of $Q$ with dimension vector $\beta$, the dimension vectors of indecomposable submodules are:

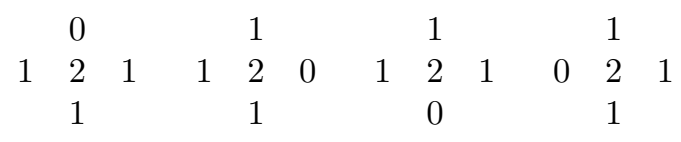

$$
\begin{aligned}
& \begin{array}{lllllllllllll} 
& 1 & & & 0 & & & 0 & & & 0 & \\
0 & 1 & 0 & 0 & 1 & 1 & 0 & 1 & 0 & 1 & 1 & 0 \\
& 0 & & & 0 & & & 1 & & & 0 &
\end{array} \\
& \begin{array}{lll} 
& 0 & \\
0 & 1 & 0 \\
& 0 &
\end{array}
\end{aligned}
$$

Let $\sigma$ be the weight given by $\sigma(\alpha)=\sum_{i=1}^{5} a_{i} \alpha(i)$, in other words

$$
\sigma=a_{4} \begin{array}{ll}
a_{1} & \\
a_{5} & a_{2} . \\
& a_{3}
\end{array}
$$

We investigate when $\operatorname{SI}(Q, \beta)_{\sigma} \neq 0$. First of all we must have $\sigma(\beta)=0$, so $a_{1}+$ $a_{2}+a_{3}+a_{4}+2 a_{5}=0$. In particular $a_{1}+a_{2}+a_{3}+a_{4}$ must be even. The 
indecomposable submodules listed above correspond to the inequalities (using $a_{5}=$ $\left.-\left(a_{1}+a_{2}+a_{3}+a_{4}\right) / 2\right)$ :

$(2)$

$$
\begin{gathered}
a_{1} \geq 0, a_{2} \geq 0, a_{3} \geq 0, a_{4} \geq 0, \\
a_{1} \leq a_{2}+a_{3}+a_{4}, a_{2} \leq a_{1}+a_{3}+a_{4}, a_{3} \leq a_{1}+a_{2}+a_{4}, a_{4} \leq a_{1}+a_{2}+a_{3}, \\
a_{1}+a_{2}+a_{3}+a_{4} \geq 0 .
\end{gathered}
$$

The last inequality is redundant.

In the next section we will see how semi-invariants can be interpreted in terms of tensor products of modules of the general linear group. This particular example shows that for a 2-dimensional vector space $U$, the tensor product of symmetric powers $S_{a_{1}}(U) \otimes S_{a_{2}}(U) \otimes S_{a_{3}}(U) \otimes S_{a_{4}}(U)$ contains a non-trivial SL $(U)$-invariant subspace if and only if $a_{1}+a_{2}+a_{3}+a_{4}$ is even and the inequalities (2) hold. In this case, the inequalities are obvious from the Clebsch-Gordan formula.

\section{Application to Littlewood-Richardson coefficients}

Let us apply Theorem [3 in the following special case. Let us define the quiver $Q=T_{n, n, n}$ as follows:

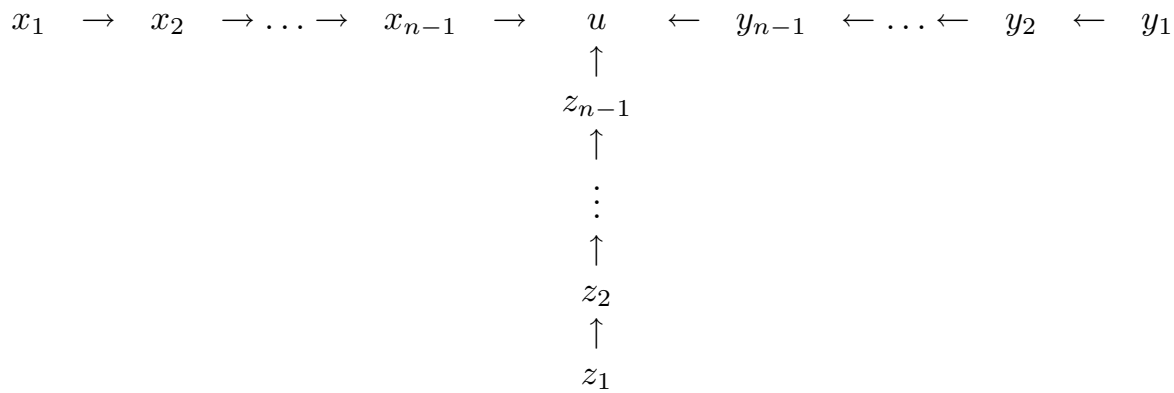

Let us choose the dimension vector $\beta\left(x_{i}\right)=\beta\left(y_{i}\right)=\beta\left(z_{i}\right)=i$ for $i=1, \ldots, n-1$, $\beta(u)=n$. The following proposition is a direct application of Cauchy's formula and is a standard calculation in representation theory.

Proposition 1. The weight space $\operatorname{SI}\left(T_{n, n, n}, \beta\right)_{\sigma}$ is isomorphic to the space of $\mathrm{SL}(U)$-invariants in the triple tensor product $S_{\lambda}(U) \otimes S_{\mu}(U) \otimes S_{\nu}(U)$ of Schur functors on $U$, where $U$ is the vector space of dimension $n$, and $\lambda, \mu, \nu$ are partitions whose conjugate partitions are given as follows:

$$
\begin{aligned}
& \lambda^{\prime}=\left((n-1)^{\sigma\left(x_{n-1}\right)},(n-2)^{\sigma\left(x_{n-2}\right)}, \ldots, 1^{\sigma\left(x_{1}\right)}\right), \\
& \mu^{\prime}=\left((n-1)^{\sigma\left(y_{n-1}\right)},(n-2)^{\sigma\left(y_{n-2}\right)}, \ldots, 1^{\sigma\left(y_{1}\right)}\right), \\
& \nu^{\prime}=\left((n-1)^{\sigma\left(z_{n-1}\right)},(n-2)^{\sigma\left(z_{n-2}\right)}, \ldots, 1^{\sigma\left(z_{1}\right)}\right) .
\end{aligned}
$$

Here $\sigma(q)$ is defined as $\sigma\left(e_{q}\right)$ where the dimension vector $e_{q}$ is given by $e_{q}(q)=1$ and $e_{q}(p)=0$ if $p \neq q$.

Proof. Let us denote by $a_{i}$ (resp. $b_{i}, c_{i}$ ) the arrow in $T_{n, n, n}$ with $t a_{i}=x_{i}, h a_{i}=x_{i+1}$ (resp. $\left.t b_{i}=y_{i}, h b_{i}=y_{i+1}, t c_{i}=z_{i}, h c_{i}=z_{i+1}\right)$ for $1 \leq i \leq n-1$. The space $\operatorname{Rep}\left(T_{n, n, n}, \beta\right)$ can be identified with

$$
\bigoplus_{1 \leq i \leq n-1}\left(\operatorname{Hom}\left(V\left(x_{i}\right), V\left(x_{i+1}\right)\right) \oplus \operatorname{Hom}\left(V\left(y_{i}\right), V\left(y_{i+1}\right)\right) \oplus \operatorname{Hom}\left(V\left(z_{i}\right), V\left(z_{i+1}\right)\right)\right)
$$

where we write $x_{n}=y_{n}=z_{n}=u$. 
The Cauchy formula [4, $\S$ A.1] gives the decomposition of $K\left[\operatorname{Rep}\left(T_{n, n, n}, \beta\right)\right]$ as a direct sum over the $3(n-1)$-tuples of partitions

$$
\left(\left(\alpha^{i}\right)_{1 \leq i \leq n-1},\left(\beta^{i}\right)_{1 \leq i \leq n-1},\left(\gamma^{i}\right)_{1 \leq i \leq n-1}\right)
$$

of the summands

$$
\bigotimes_{1 \leq i \leq n-1}\left(S_{\alpha^{i}} V\left(x_{i}\right) \otimes S_{\alpha^{i}} V\left(x_{i+1}\right)^{*} \otimes S_{\beta^{i}} V\left(y_{i}\right) \otimes S_{\beta^{i}} V\left(y_{i+1}\right)^{*}\right.
$$

$$
\left.\otimes S_{\gamma^{i}} V\left(z_{i}\right) \otimes S_{\gamma^{i}} V\left(z_{i+1}\right)^{*}\right) .
$$

Let us denote $H=\prod_{1 \leq i \leq n-1}\left(\operatorname{SL}\left(V\left(x_{i}\right)\right) \times \mathrm{SL}\left(V\left(y_{i}\right)\right) \times \mathrm{SL}\left(V\left(z_{i}\right)\right)\right)$. Then it follows from the Littlewood-Richardson Rule [4, $\S$ A.1] that the summand corresponding to the $3(n-1)$-tuple

$$
\left(\left(\alpha^{i}\right)_{1 \leq i \leq n-1},\left(\beta^{i}\right)_{1 \leq i \leq n-1},\left(\gamma^{i}\right)_{1 \leq i \leq n-1}\right)
$$

contains an $H$-invariant if and only if we have for each $i, 1 \leq i \leq n-1$,

$$
\begin{aligned}
& \left(\alpha^{i}\right)^{\prime}=\left((i)^{\sigma\left(x_{i}\right)},(i-1)^{\sigma\left(x_{i-1}\right)}, \ldots, 1^{\sigma\left(x_{1}\right)}\right), \\
& \left(\beta^{i}\right)^{\prime}=\left((i)^{\sigma\left(y_{i}\right)},(i-1)^{\sigma\left(y_{i-1}\right)}, \ldots, 1^{\sigma\left(y_{1}\right)}\right), \\
& \left(\gamma^{i}\right)^{\prime}=\left((i)^{\sigma\left(z_{i}\right)},(i-1)^{\sigma\left(z_{i-1}\right)}, \ldots, 1^{\sigma\left(z_{1}\right)}\right)
\end{aligned}
$$

for some non-negative numbers $\sigma\left(x_{i}\right), \sigma\left(y_{i}\right), \sigma\left(z_{i}\right)$. Moreover, if these conditions are satisfied, then the space of $H$-invariants is isomorphic to

$$
S_{\alpha^{n-1}} V(u)^{*} \otimes S_{\beta^{n-1}} V(u)^{*} \otimes S_{\gamma^{n-1}} V(u)^{*} .
$$

Therefore the space of $\mathrm{SL}\left(T_{n, n, n}, \beta\right)$-semi-invariants can be identified with the space of $\mathrm{SL}(V(u))$-invariants in the above triple tensor product.

Corollary 2. The set of triples of partitions $(\lambda, \mu, \nu)$ such that the space of $\mathrm{SL}(U)$ invariants in $S_{\lambda}(U) \otimes S_{\mu}(U) \otimes S_{\nu}(U)$ is non-zero, in the space of triples of weights is given by a finite set of linear homogeneous inequalities in the parts of $\lambda, \mu, \nu$ and the condition that $|\lambda|+|\mu|+|\nu|$ is divisible by $n:=\operatorname{dim} U$.

Proof. Let $\sigma \in \Gamma$ be given by (3) and let $\sigma(\beta)=0$. All components of $\sigma$ are integers only if $|\lambda|+|\mu|+|\nu|$ is divisible by $n$, because

$$
0=\sigma(\beta)=n \sigma(u)+\sum_{i=1}^{n-1} i\left(\sigma\left(x_{i}\right)+\sigma\left(y_{i}\right)+\sigma\left(z_{i}\right)\right)=n \sigma(u)+|\lambda|+|\mu|+|\nu| .
$$

By Theorem 3 and Proposition 11 those $(\lambda, \mu, \nu)$ for which $\operatorname{SI}\left(T_{n, n, n}, \beta\right)_{\sigma} \neq 0$ are given by $\sigma(\beta)=0$ and a finite set of homogeneous linear inequalities in $\sigma\left(x_{i}\right), \sigma\left(y_{i}\right), \sigma\left(z_{i}\right), 1 \leq i \leq n-1$. These inequalities can be written as inequalities in the parts of $\lambda, \mu$ and $\nu$.

\section{The Proof of Theorem 1}

We define $[x, y]$ to be the vector space with the basis formed by paths from $x$ to $y$. We assumed that $Q$ has no oriented cycles, so the spaces $[x, y]$ are finite dimensional.

The indecomposable projective representations are in a bijection with $Q_{0}$. The indecomposable projective corresponding to $x$ is defined by

$$
P_{x}(y)=[x, y], \quad P_{x}(a)=a \circ \cdot:[x, t a] \rightarrow[x, h a],
$$


where $P_{x}(a)$ is given by the composition $p \mapsto a \circ p$. We have $\operatorname{Hom}_{Q}\left(P_{x}, V\right)=V(x)$. In particular $\operatorname{Hom}_{Q}\left(P_{x}, P_{y}\right)=[y, x]$.

We choose a numbering $Q_{0}=\left\{x_{1}, \ldots, x_{n}\right\}$ of vertices of $Q$ such that for every $\alpha \in Q_{1}$ with $t \alpha=x_{i}, h \alpha=x_{j}$, we have $i<j$. Let $b_{i, j}$ be the number of arrows $\alpha \in Q_{1}$ with $t \alpha=x_{i}, h \alpha=x_{j}$. Let $p_{i, j}=\operatorname{dim}\left[x_{i}, x_{j}\right]$ be the number of paths $p$ in $Q$ such that $t p=x_{i}, h p=x_{j}$.

The relations between the $\alpha\left(x_{j}\right)$ and $\sigma\left(x_{i}\right)$ are as follows:

$$
\begin{gathered}
\sigma\left(x_{j}\right)=\alpha\left(x_{j}\right)-\sum_{i<j} b_{i, j} \alpha\left(x_{i}\right), \\
\alpha\left(x_{j}\right)=\sigma\left(x_{j}\right)+\sum_{i<j} p_{i, j} \sigma\left(x_{i}\right) .
\end{gathered}
$$

We define the $m$-arrow quiver $\Theta_{m}$ as a quiver with two vertices $x_{+}$and $x_{-}$, and $m$ arrows $a_{1}, \ldots, a_{m}$ with $t a_{i}=x_{-}, h a_{i}=x_{+}$for $i=1, \ldots, m$. We define the weight $\tau$ given by $\tau\left(x_{+}\right)=1, \tau\left(x_{-}\right)=-1$. The dimension vector $\theta(n)$ is defined by $\theta(n)\left(x_{+}\right)=\theta(n)\left(x_{-}\right)=n$.

The idea of the proof of Theorem 1 is to reduce the calculation to the weight space $\operatorname{SI}\left(\Theta_{m}, \theta(n)\right)_{\tau}$. The method comes from Classical Invariant Theory with a slight adjustment to accomodate the definition of semi-invariants $c^{V}$.

Proof of Theorem 1 Let us fix $Q, \beta$ and a weight $\sigma$. We proceed in three steps. In the first step, we reduce the theorem to the case that $Q$ is a quiver with exactly one source $x_{-}$and one sink $x_{+}$, and $\sigma\left(x_{-}\right)=1, \sigma\left(x_{+}\right)=-1$ and $\sigma$ is zero on all other vertices. In the second step we reduce to the case that there are no vertices $x$ with $\sigma(x)=0$. The only case left is the quiver $\Theta_{m}$ with weight $\tau$. In Step 3 we will prove the theorem in this case.

Step 1. Construct a quiver $Q(\sigma)$ as follows:

$$
\begin{aligned}
& Q(\sigma)_{0}=Q_{0} \cup x_{-} \cup x_{+}, \\
& Q(\sigma)_{1}=Q_{1} \cup Q_{-} \cup Q_{+}
\end{aligned}
$$

where $Q_{-}$consists of the set of arrows from $x_{-}$to $x_{i}$, with $\sigma\left(x_{i}\right)$ arrows going to the vertex $x_{i}$ for which $\sigma\left(x_{i}\right)>0$ and no arrows going to other vertices. The set $Q_{+}$consists of the set of arrows from $x_{i}$ to $x_{+}$, with $-\sigma\left(x_{i}\right)$ arrows going from the vertex $x_{i}$ for which $\sigma\left(x_{i}\right)<0$ and no arrows going from other vertices to $x_{+}$.

Example 2. Let $Q$ be the quiver

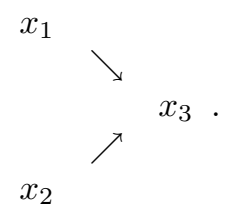

Let $\sigma=(1,1,-2)$. Then the quiver $Q(\sigma)$ is

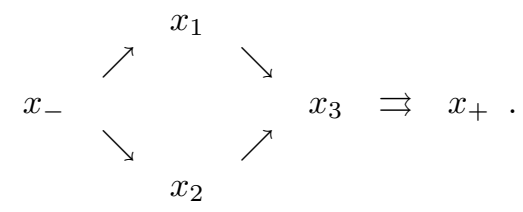


We will write $\bar{Q}=Q(\sigma)$. Define the weight $\bar{\sigma}$ of $\bar{Q}$ by $\bar{\sigma}\left(x_{-}\right)=1, \bar{\sigma}\left(x_{i}\right)=$ $0, \bar{\sigma}\left(x_{+}\right)=-1$. The dimension vector $\bar{\beta}=\beta(\sigma)$ is defined by $\bar{\beta}\left(x_{i}\right)=\beta\left(x_{i}\right)$, $\bar{\beta}\left(x_{-}\right)=\sum_{\left\{i \mid \sigma\left(x_{i}\right)>0\right\}} \sigma\left(x_{i}\right) \beta\left(x_{i}\right), \bar{\beta}\left(x_{+}\right)=\sum_{\left\{i \mid \sigma\left(x_{i}\right)<0\right\}}-\sigma\left(x_{i}\right) \beta\left(x_{i}\right)$. Suppose that $W \in \operatorname{Rep}(\bar{Q}, \bar{\beta})$. The matrices of all maps $W(a)$ with $a \in Q_{-}$form a square matrix. Let $D^{-}(W)$ be the determinant of this block matrix. Let $D^{+}(W)$ be the determinant of all $W(a)$ with $a \in Q_{+}$. Then the correspondence $c \rightarrow D^{-} c D^{+}$gives the isomorphism of weight spaces $\operatorname{SI}(Q, \beta)_{\sigma} \rightarrow \operatorname{SI}(\bar{Q}, \bar{\beta})_{\bar{\sigma}}$.

Let $\bar{\alpha}$ be the dimension vector of $\bar{Q}$ such that $\bar{\sigma}=\langle\bar{\alpha}, \cdot\rangle$. Let $\bar{V}$ be a representation of $\bar{Q}$ with dimension vector $\bar{\alpha}$ and let $c^{\bar{V}}$ be the corresponding non-zero semi-invariant on $\operatorname{SI}(\bar{Q}, \bar{\beta})$.

Proposition 2. The factor $c$ in the decomposition $c^{\bar{V}}=D^{-} c D^{+}$is of the form $c^{V}$ for some $V \in \operatorname{Rep}(Q, \alpha)$.

Proof. Notice that the weight of $D^{-}$is equal to $\left\langle\gamma_{-}, \cdot\right\rangle$ where

$$
\gamma_{-}\left(x_{-}\right)=1, \quad \gamma_{-}\left(x_{j}\right)=\gamma_{-}\left(x_{+}\right)=0 .
$$

Similarly, by (15), the weight of $D^{+}$equals $\left\langle\gamma_{+}, \cdot\right\rangle$ where

$$
\begin{gathered}
\gamma_{+}\left(x_{-}\right)=0, \quad \gamma_{+}\left(x_{j}\right)=-\sum_{\substack{i \leq j \\
\sigma\left(x_{i}\right)<0}} p_{i, j} \sigma\left(x_{i}\right), \\
\gamma_{+}\left(x_{+}\right)=-1+\sum_{\substack{j \\
\sigma\left(x_{j}\right)<0}} \sum_{\substack{i \leq j \\
\sigma\left(x_{i}\right)<0}} p_{i, j} \sigma\left(x_{i}\right) \sigma\left(x_{j}\right) .
\end{gathered}
$$

It is easy to see that $\left\langle\gamma_{-}, \bar{\beta}\right\rangle=\left\langle\gamma_{+}, \bar{\beta}\right\rangle=0$.

Let $\bar{V} \in \operatorname{Rep}(\bar{Q}, \bar{\alpha})$. Then $\bar{V}$ has an obvious submodule $\bar{V}_{1}=\left.\bar{V}\right|_{\bar{Q}_{0} \backslash\left\{x_{-}\right\}}$. We have an exact sequence

$$
0 \rightarrow \bar{V}_{1} \rightarrow \bar{V} \rightarrow \bar{V}_{2} \rightarrow 0
$$

with the dimension of $\bar{V}_{2}$ equal to $\gamma_{-}$.

Let $M$ be the module defined by the exact sequence

$$
0 \rightarrow P_{x_{+}} \stackrel{i}{\rightarrow} \bigoplus_{b, h b=x_{+}} P_{t b} \rightarrow M \rightarrow 0
$$

where the morphism $i$ from $P_{x_{+}}$to a copy $P_{t b}$ maps the trivial path $e\left(x_{+}\right)$to the path $b$. The dimension vector of $M$ is $\gamma_{+}$, and $c^{M}$ is the determinant $D^{+}$. Consider the map

$$
\sum_{\substack{b \\ h b=x_{+}}} \bar{V}_{1}(b): \bigoplus_{b, h b=x_{+}} \bar{V}_{1}(t b) \rightarrow \bar{V}_{1}\left(x_{+}\right) .
$$

The dimension of the kernel is at least 1 . Let $\left(s_{b}\right)_{b, h b=x_{+}}$with $s_{b} \in \bar{V}_{1}(t b)$ be a non-trivial element in the kernel. We can now define a map $\bigoplus_{b, h b=x_{+}} P_{t b} \rightarrow \bar{V}_{1}$ by sending the generator $e(t b) \in P_{t b}(t b)$ to $s_{b}$ for all $b$. Because $\left(s_{b}\right)_{b, h b=x_{+}}$lies in the kernel, this actually defines a morphism $M \rightarrow \bar{V}_{1}$. Let $\bar{V}_{3}$ be the image of this morphism.

Now $\bar{V}_{3}$ is a submodule of $\bar{V}_{1}$ and $c^{\bar{V}_{1}} \neq 0$. By Lemma[1a) we have $\left\langle\underline{d}\left(\bar{V}_{3}\right), \bar{\beta}\right\rangle \geq$ 0 . We also have $c^{M}=D^{+} \neq 0$. If we apply Lemma 1 a) to the kernel $N$ of 
$M \rightarrow \bar{V}_{3}$, then we get $\langle\underline{d}(N), \bar{\beta}\rangle=\left\langle\gamma_{+},-\underline{d}\left(\bar{V}_{3}\right)\right\rangle=-\left\langle\underline{d}\left(\bar{V}_{3}\right), \bar{\beta}\right\rangle \geq 0$. We conclude that $\left\langle\underline{d}\left(\bar{V}_{3}\right), \bar{\beta}\right\rangle=0$. By Lemma 1 b) $c^{\bar{V}_{3}}$ divides the semi-invariant $c^{M}=D^{+}$. Because $D^{+}$is an irreducible semi-invariant we must have $c^{\bar{V}_{3}}=D^{+}, \gamma_{+}=\operatorname{dim} \bar{V}_{3}$ and $\bar{V}_{3}$ is isomorphic to $M$.

We have an exact sequence

$$
0 \rightarrow \bar{V}_{3} \rightarrow \bar{V}_{1} \rightarrow \bar{V}_{4} \rightarrow 0
$$

Now it is clear by the multiplicative property that $c^{\bar{V}}=c^{\bar{V}_{2}} c^{\bar{V}_{4}} c^{\bar{V}_{3}}$ with the first factor being proportional to $D^{-}$and the last one to $D^{+}$. Let us also define a submodule $\bar{V}_{5}=\left.\bar{V}_{4}\right|_{\left\{x_{+}\right\}}$, so we have an exact sequence

$$
0 \rightarrow \bar{V}_{5} \rightarrow \bar{V}_{4} \rightarrow \bar{V}_{6} \rightarrow 0
$$

Note that $\bar{V}_{6}$ has support within $Q$. The restriction of $\bar{V}_{6}$ to $Q$ will be denoted by $V$. We will prove that the restriction of $c^{\hat{V}}$ to $\operatorname{Rep}(Q, \beta)$ is $c^{V}$.

Extend $W \in \operatorname{Rep}(Q, \beta)$ to the module $\bar{W}$ of dimension $\bar{\beta}$ by putting $\bar{W}\left(x_{-}\right)=$ $\bigoplus_{a, t a=x_{-}} W(h a), \bar{W}\left(x_{+}\right)=\bigoplus_{b, h b=x_{+}} W(t b)$, with the maps $\bar{W}(a)$ and $\bar{W}(b)$ being the components of the identity map. Define the canonical submodule $\bar{W}_{1}=$ $\left.\bar{W}\right|_{\left\{x_{+}\right\}}$. We have an exact sequence

$$
0 \rightarrow \bar{W}_{1} \rightarrow \bar{W} \rightarrow \bar{W}_{2} \rightarrow 0
$$

Define the submodule $\bar{W}_{3}=\left.\bar{W}_{2}\right|_{\hat{Q} \backslash\left\{x_{-}\right\}}$of $\bar{W}_{2}$. Now we have an exact sequence

$$
0 \rightarrow \bar{W}_{3} \rightarrow \bar{W}_{2} \rightarrow \bar{W}_{4} \rightarrow 0
$$

The representation $\bar{W}_{3}$ has support within $Q$ and its restriction to $Q$ is just $W$.

We now have

$$
c^{\bar{V}}(\bar{W})=c^{\bar{V}_{4}}(\bar{W})=c^{\bar{V}_{4}}\left(\bar{W}_{1}\right) c^{\bar{V}_{4}}\left(\bar{W}_{3}\right) c^{\bar{V}_{4}}\left(\bar{W}_{4}\right)=c^{\bar{V}_{4}}\left(\bar{W}_{3}\right)
$$

because $c^{\bar{V}_{4}}\left(\bar{W}_{1}\right)$ and $c^{\bar{V}_{4}}\left(\bar{W}_{4}\right)$ are constant. Moreover,

$$
c^{\bar{V}_{4}}\left(\bar{W}_{3}\right)=c^{\bar{V}_{5}}\left(\bar{W}_{3}\right) c^{\bar{V}_{6}}\left(\bar{W}_{3}\right)=c^{\bar{V}_{6}}\left(\bar{W}_{3}\right)=c^{V}(W)
$$

because $c^{\bar{V}_{5}}\left(\bar{W}_{4}\right)$ is constant. This concludes the proof of the proposition.

Step 2. Let $Q, \beta, \sigma$ be as above. Let $x \in Q_{0}$ be a vertex such that $\sigma(x)=0$. Let $a_{1}, \ldots, a_{s}$ be the arrows in $Q_{1}$ with $h a_{k}=x(k=1, \ldots, s)$ and let $b_{1}, \ldots, b_{t}$ be the arrows in $Q_{1}$ with $t b_{l}=x(l=1, \ldots, t)$. Let $\bar{Q}$ be the quiver such that $\bar{Q}_{0}=Q_{0} \backslash\{x\}$ and $\bar{Q}_{1}=\left(Q_{1} \backslash\left\{a_{1}, \ldots, a_{s}, b_{1}, \ldots, b_{t}\right\}\right) \cup\left\{b a_{k, l}\right\}_{1 \leq k \leq s, 1 \leq l \leq t}$, where $t\left(b a_{k, l}\right)=t a_{k}, h\left(b a_{k, l}\right)=h b_{l}$. Let $\bar{\beta}, \bar{\sigma}$ be the restrictions of $\beta, \sigma$ to $Q_{0} \backslash\{x\}$.

The Fundamental Theorem of Invariant Theory (see 2 for a characteristic free version) says that every semi-invariant from $\operatorname{SI}(Q, \beta)_{\sigma}$ can be obtained from the semi-invariants from $\operatorname{SI}(\bar{Q}, \bar{\beta})_{\bar{\sigma}}$ by substituting the actual compositions $b_{l} a_{k}$ for the arrows of type $b a_{k, l}$. Assuming Theorem 1 for $\operatorname{SI}(\bar{Q}, \bar{\beta})_{\bar{\sigma}}$ to be true, we need to show that every semi-invariant $c^{\bar{V}}$ from $\operatorname{SI}(\bar{Q}, \bar{\beta})_{\bar{\sigma}}$ pulls back to a semi-invariant of type $c^{V}$. For a given representation $\bar{V}$ of $\bar{Q}$ of dimension $\bar{\alpha}$ we define the representation $V=$ ind $\bar{V}$ as follows. We notice that the condition $\sigma(x)=0$ means that we expect $\operatorname{dim} V(x)=\sum_{k=1}^{s} \operatorname{dim} V\left(t a_{k}\right)$. 
This means we put

$$
V(y)= \begin{cases}\bar{V}(y) & \text { if } y \neq x \\ \bigoplus_{k=1}^{s} \bar{V}\left(t a_{k}\right) & \text { if } y=x\end{cases}
$$

We define the linear maps $V(a)$ as follows:

$$
V(a)= \begin{cases}\bar{V}(a) & \text { if } a \neq a_{k}, b_{l}, \\ i\left(a_{k}\right) & \text { if } a=a_{k}, \\ \sum_{k=1}^{s} \bar{V}\left(b a_{k, l}\right) & \text { if } b=b_{l},\end{cases}
$$

where $i\left(a_{k}\right): V\left(t a_{k}\right) \rightarrow \bigoplus_{k=1}^{s} V\left(t a_{k}\right)$ is the injection on the $k$-th summand.

Then it is easy to check directly from the definition of semi-invariants $c^{V}$ that if the representation $\bar{W}=$ res $W$ of dimension $\bar{\beta}$ is a restriction of a representation $W$ of $Q$ of dimension $\beta$, then $c^{\bar{V}}(\bar{W})=c^{V}(W)$.

Notice that the functor ind $\bar{V}$ is the left adjoint of the obvious restriction functor res : $\operatorname{Rep}(Q) \rightarrow \operatorname{Rep}(\bar{Q})$, i.e., we have the natural isomorphisms

$$
\operatorname{Hom}_{Q}(\text { ind } \bar{V}, W)=\operatorname{Hom}_{\bar{Q}}(\bar{V} \text {, res } W)
$$

which explains why $c^{\bar{V}}(\bar{W})$ and $c^{V}(W)$ vanish simultaneously.

Step 3. It remains to deal directly with the weight space $\operatorname{SI}\left(\Theta_{m}, \theta(n)\right)_{\tau}$. Writing the representation $W$ of dimension $\theta(n)$ as an $m$-tuple of linear maps,

$$
W\left(a_{1}\right), \ldots, W\left(a_{m}\right): W_{-} \rightarrow W_{+},
$$

we can introduce the additional action of the group $\mathrm{GL}(m)$ acting on this space by taking linear combinations of the linear maps $W\left(a_{1}\right), \ldots, W\left(a_{m}\right)$. Using the Cauchy formula (in its characteristic free version, say from [1]) we see that the space $\operatorname{SI}\left(\Theta_{m}, \theta(n)\right)_{\tau}$ of semi-invariants can be identified with $\bigwedge^{n} W_{-} \otimes \bigwedge^{n} W_{+}^{*} \otimes D_{n}\left(K^{m}\right)$. Here $D_{n}$ denotes the $n$-th divided power. Since the divided power $D_{n}\left(K^{m}\right)$ is generated as a $\mathrm{GL}(m)$-module by its highest weight vector (which corresponds to the semi-invariant det $\left.W\left(a_{1}\right)\right)$ and the set of semi-invariants of the form $c^{V}$ is preserved by the action of $\mathrm{GL}(m)$, it is enough to express $\operatorname{det} W\left(a_{1}\right)$ as the semi-invariant of the form $c^{V}$. Notice that $\tau=\langle\alpha, \cdot\rangle$ for the dimension vector $\alpha=(1, m-1)$. Taking the module $V$ to be the $m$-tuple of linear maps $V\left(a_{1}\right), \ldots, V\left(a_{m}\right): K \rightarrow K^{m-1}$ where $V\left(a_{1}\right)=0$ and $V\left(a_{i}\right)$ is the embedding sending 1 to the $i-1$ 'st basis vector, for $i=2, \ldots, m$, we check directly that $c^{V}=\operatorname{det} W\left(a_{1}\right)$. This concludes the proof of Theorem 1 .

We now will give another description for semi-invariants $\operatorname{SI}(Q, \beta)_{\sigma}$. Let $\bar{Q}=$ $Q(\sigma), \bar{\beta}$ and $\bar{\sigma}$ be as in Step 1 of the proof of Theorem 1. We know that $\operatorname{SI}(Q, \beta)_{\sigma} \cong$ $\operatorname{SI}(\bar{Q}, \bar{\beta})_{\bar{\sigma}}$. Let $\bar{\alpha}$ be a dimension vector of $\bar{Q}$ such that $\langle\bar{\alpha}, \cdot\rangle=\bar{\sigma}$. Now $\operatorname{SI}(\bar{Q}, \bar{\beta})_{\bar{\sigma}}$ is generated by semi-invariants $c^{\bar{V}}$ with $\underline{d}(\bar{V})=\bar{\alpha}$. In fact we only need to take those $c^{\bar{V}}$ where $\bar{V}$ lies in a Zariski dense set of $\operatorname{Rep}(\bar{Q}, \bar{\alpha})$. A general representation $\bar{V}$ of dimension $\bar{\alpha}$ has the following projective resolution:

$$
0 \rightarrow P_{x_{+}} \stackrel{d_{V}}{\longrightarrow} P_{x_{-}} \rightarrow \bar{V} \rightarrow 0
$$

with $d_{V} \in \operatorname{Hom}_{Q}\left(P_{x_{+}}, P_{x_{-}}\right)=\left[x_{-}, x_{+}\right]$. So $d_{V}$ can be seen as some linear combination $\sum_{i=1}^{r} \lambda_{i} p_{i}$ where $p_{1}, \ldots, p_{r}$ are all paths from $x_{+}$to $x_{-}$. For any $\bar{W} \in \operatorname{Rep}(\bar{Q}, \bar{\beta})$ we have the following exact sequence:

$$
0 \rightarrow \operatorname{Hom}_{\bar{Q}}(\bar{V}, \bar{W}) \rightarrow \operatorname{Hom}_{\bar{Q}}\left(P_{x_{+}}, \bar{W}\right) \stackrel{\tilde{d}_{\bar{V}}}{\longrightarrow} \operatorname{Hom}_{\bar{Q}}\left(P_{x_{-}}, \bar{W}\right) \rightarrow \operatorname{Ext}_{\bar{Q}}(\bar{V}, \bar{W}) \rightarrow 0 .
$$


It is easy to see that $\operatorname{det}\left(\tilde{d}_{\bar{V}}\right)=c^{\bar{V}}(\bar{W})=c^{V}(W)$.

We have that

$$
\begin{aligned}
& \operatorname{Hom}_{\bar{Q}}\left(P_{x_{+}}, \bar{W}\right) \cong \bar{W}_{x_{+}}=\bigoplus_{\sigma\left(x_{i}\right)>0} W\left(x_{i}\right)^{\sigma\left(x_{i}\right)} \\
& \operatorname{Hom}_{\bar{Q}}\left(P_{x_{-}}, \bar{W}\right) \cong \bar{W}_{x_{-}}=\bigoplus_{\sigma\left(x_{i}\right)<0} W\left(x_{i}\right)^{\sigma\left(x_{i}\right)} \\
& \tilde{d}_{\bar{V}}=\sum_{i} \lambda_{i} \bar{V}\left(p_{i}\right) .
\end{aligned}
$$

Let $F$ be a function from the set of paths from $x_{+}$to $x_{-}$to the set of non-negative integers. For each such $F$ we can define the semi-invariant $I_{F}$ as the coefficient of $\lambda_{1}^{F\left(p_{1}\right)} \lambda_{2}^{F\left(p_{2}\right)} \ldots \lambda_{r}^{F\left(p_{r}\right)}$ in $\operatorname{det}\left(\tilde{d}_{\bar{V}}\right)$.

Corollary 3. The space of semi-invariants $\operatorname{SI}(Q, \beta)_{\sigma}$ is spanned by semi-invariants of the form $I_{F}$.

A necessary condition for $I_{F}$ to be non-zero is

$$
\sum_{i} F\left(p_{i}\right)=\sum_{\sigma\left(x_{i}\right)>0} \sigma\left(x_{i}\right) \beta\left(x_{i}\right)=\sum_{\sigma\left(x_{i}\right)<0}-\sigma\left(x_{i}\right) \beta\left(x_{i}\right) .
$$

\section{ACKNOWLEDGEMENT}

The authors would like to thank Andrei Zelevinsky for helpful suggestions.

\section{REFERENCES}

[1] K. Akin, D. A. Buchsbaum, J. Weyman, Schur functors and Schur complexes, Adv. Math. 44 (1982), 207-278. MR 84c:20021

[2] C. DeConcini, C. Procesi, Characteristic free approach to invariant theory, Adv. Math. 21 (1976), 330-354. MR 54:10305

[3] W. Fulton, Eigenvalues of sums of Hermitian matrices (after A. Klyachko), Séminaire Bourbaki (1998). MR 99m:00026

[4] W. Fulton, J. Harris, Representation Theory, Springer-Verlag, New York, 1991. MR 93a:20069

[5] V. Kac, Infinite root systems, representations of graphs and invariant theory II, J. Algebra 78 (1982), 141-162. MR 85b:17003

[6] A. D. King, Moduli of representation of finite dimensional algebras, Quart. J. Math. Oxford (2) 45 (1994), 515-530. MR 96a:16009

[7] A. Klyachko, Stable vector bundles and Hermitian operators, IGM, University of Marne-laVallee, preprint (1994).

[8] A. Knutson, T. Tao, The honeycomb model of $\mathrm{GL}_{n}(\mathbb{C})$ tensor products, I: Proof of the saturation conjecture, J. Amer. Math. Soc. 12 (1999), 1055-1090. MR 2000c:20066

[9] C.M. Ringel, Representations of K-species and bimodules, J. Algebra 41 (1976) 269-302. MR 54:10340

[10] A. Schofield, Semi-invariants of quivers, J. London Math. Soc. 43 (1991), 383-395. MR 92g:16019

[11] A. Schofield, General representations of quivers, Proc. London Math. Soc. (3) 65 (1992) 46-64. MR 93d:16014

[12] A. Schofield, M. van den Bergh, Semi-invariants of quivers for arbitrary dimension vectors, preprint, math.RA/9907174.

Department of Mathematics, Massachusetts Institute of Technology, Cambridge, MASSACHUSETTS 02151

E-mail address: hderksen@math.mit.edu

Department of Mathematics, Northeastern University, Boston, Massachusetts 02115

E-mail address: weyman@neu.edu 